\title{
Matching of turn pattern measurements for cyclotrons using multiobjective optimization
}

\author{
Matthias Frey, ${ }^{*}$ Jochem Snuverink, ${ }^{\dagger}$ Christian Baumgarten, ${ }^{*}$ and Andreas Adelmann ${ }^{\S}$ \\ Paul Scherrer Institut (PSI), CH-5232 Villigen PSI, Switzerland
}

(Received 22 March 2019; published 24 June 2019; corrected 1 December 2020)

\begin{abstract}
The usage of numerical models to study the evolution of particle beams is an essential step in the design process of particle accelerators. However, uncertainties of input quantities such as the beam energy and magnetic field lead to simulation results that do not fully agree with measurements; hence, the final machine will behave slightly differently than the simulations. In the case of cyclotrons, such discrepancies affect the overall turn pattern or may even alter the number of turns in the machine. Inaccuracies at the PSI Ring cyclotron facility that may harm the isochronism are compensated by additional magnetic fields provided by 18 trim coils. These are often absent from simulations or their implementation is very simplistic. In this paper, a newly developed realistic trim coil model within the particle accelerator framework OPAL is presented that was used to match the turn pattern of the PSI Ring cyclotron. Because of the high-dimensional search space consisting of 48 design variables (simulation input parameters) and 182 objectives (i.e., turns), the simulation and measurement cannot be matched in a straightforward manner. Instead, an evolutionary multiobjective optimization with a population size of more than 8000 individuals per generation together with a local search approach were applied that reduced the maximum absolute error to $4.5 \mathrm{~mm}$ over all 182 turns.
\end{abstract}

DOI: $10.1103 /$ PhysRevAccelBeams.22.064602

\section{INTRODUCTION}

The PSI Ring cyclotron was commissioned in the mid1970s and has been in user operation since. It has a long history of upgrades and improvements that made it possible to operate the machine with currents of up to $2.4 \mathrm{~mA}$, a figure exceeding the design specification by a factor of 24 . But the operation and development of accelerators over several decades is challenging in many ways. Keeping documentation up to date has been proven very challenging and in some cases even impossible. Beam lines or insertion devices have been modified or replaced, and sputtering processes change the form of collimators and apertures. However, a postcommissioning survey of possibly activated accelerator components is difficult and risky, as it requires a partial or total disassembly; hence, components may not be accessible with reasonable effort. Thus, it is no surprise that the work to improve, optimize, replace, or test

\footnotetext{
*matthias.frey@psi.ch

jochem.snuverink@psi.ch

christian.baumgarten@psi.ch

§andreas.adelmann@psi.ch
}

Published by the American Physical Society under the terms of the Creative Commons Attribution 4.0 International license. Further distribution of this work must maintain attribution to the author(s) and the published article's title, journal citation, and DOI. models of accelerators continues even after decades of successful operation.

Here we report our efforts to model and fit the turn pattern of the PSI Ring cyclotron, for which accurate magnetic field data of the trim coil fields are not available. Instead, the average field profiles of the trim coils are derived from measurements of beam phase shifts. If a local field change by some inner trim coil causes a local radial shift of some turn, this subsequently leads, in combination with a slight difference in the betatron tune, phase, and amplitude, to a significant difference in the overall turn pattern. This becomes more and more significant from turn to turn. Hence, the overall fit should be most sensitive to the innermost trim coils.

Besides the contributions of the trim coils to the total magnetic field, the accuracy of the voltage profiles of the rf resonators plays a crucial role for the exact form of the turn pattern, too. The PSI Ring cyclotron is equipped with five rf cavities, i.e., four main cavities and a third harmonic flattop cavity (see Fig. 1). While all main cavities could, in principle, have the same field profiles, they are not always operated at the exact same voltage. Furthermore, due to the sheer size of the Ring cyclotron, the exact position of the cavities might slightly differ from one to another. Hence, a fit of the turn pattern in the Ring must very likely allow for (small) variations of the positions and voltages of the cavities.

Various computer codes are able to compute turn patterns of cyclotrons. A survey of the most common 


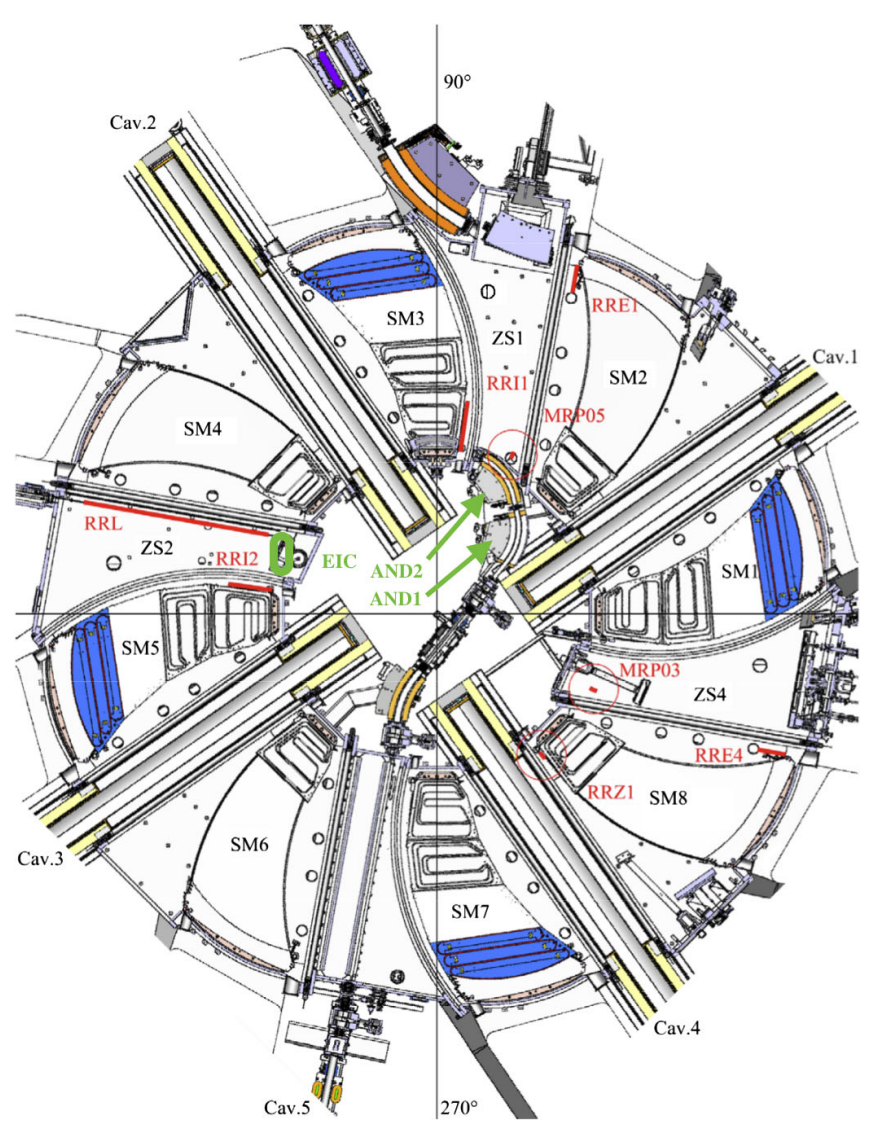

FIG. 1. Plan of the PSI Ring cyclotron. Probes and monitors are marked in red [1]. Injection elements are marked in green.

cyclotron codes is given in Ref. [2]. A first step towards a realistic numerical model of the PSI Ring cyclotron using OPAL was taken in Ref. [3], but only the last few turns before extraction and one of the 18 trim coils were included. Another preceding study [4] has shown, however, that it is a challenging task to match all 182 turns and likely requires more free parameters with at least all trim coil amplitudes, but also cavity voltages and possibly cavity alignment errors.

Because of the large design and objective spaces, a simple parameter tweaking by hand is infeasible. A remedy is the use of global optimization techniques. In this paper, an available framework of an evolutionary algorithm is applied that is complemented with a local search. Several papers such as Refs. [5-11] already showed the successful application of evolutionary algorithms like particle swarm, differential evolution, or nondominated sorting genetic algorithm (NSGA-II) [12] in connection with particle accelerator modeling.

In this paper, a general trim coil model is presented that is integrated into the particle accelerator framework OPAL [13]. It allows a more realistic description of the magnetic fields based on measured data. Together with the builtin multiobjective genetic algorithm (MOGA) and a local search, it was possible to match all turns of the PSI Ring cyclotron to a maximum absolute error of $4.5 \mathrm{~mm}$. With the exception of Refs. [4,14], the authors are not aware of any paper that tries to match the measured turn pattern with a simulation in the context of cyclotrons.

The paper is structured as follows: In Sec. II, the aforementioned cyclotron is described, and the new trim coil model is explained before the next section discusses its modeling and implementation within OPAL. The results of both approaches, i.e., local search and MOGA, are shown in Sec. IV with a closer discussion in Sec. V. Final remarks and a conclusion are gathered in the last section.

\section{PSI RING CYCLOTRON}

Figure 1 shows the eight-sector (SM1-SM8) Ring cyclotron at PSI that is the last accelerating stage of the High Intensity Proton Accelerator (HIPA) facility accelerating routinely $2.2 \mathrm{~mA}$ (maximum $2.4 \mathrm{~mA}$ ) proton beams with the four main cavities (cavities 1-4) and one flattop cavity (cavity 5) at $50.65 \mathrm{MHz}$ from 72 to $590 \mathrm{MeV}$. A beam is injected at an azimuth of $110^{\circ}$ and a radius around $2 \mathrm{~m}$. After typically 182 turns, it is extracted and guided to several targets to produce either muons (through pion decay) or neutrons.

\section{A. Radial probes}

The cyclotron is equipped with a total of five wire probes that enable beam profiles [1]. However, here we use only data of the probes RRI2 (turns 1-16) and RRL (turns 9-182).

The beam profiles are obtained by measuring the current of the wire while it moves radially through the median plane and crosses subsequently multiple turns. The step width of the RRI 2 and RRL probes are 0.1 and $0.5 \mathrm{~mm}$, respectively. The RRL probe has a single vertical carbon wire, while the RRI2 probe has three carbon wires, two crossed and one vertical, the combination of which enables one to obtain information about the shape and vertical position of the beam. Here we use exclusively information of the vertical wires. Examples of the (normalized) profile measurement for the RRI2 and RRL probes are given in Figs. 3 and 4, respectively. The wire position, relative to the machine center at $(0,0)$, is described by (cf. Fig. 2)

$$
\left(\begin{array}{l}
x \\
y
\end{array}\right)=s \cdot\left(\begin{array}{c}
\cos (\varphi) \\
\sin (\varphi)
\end{array}\right)+\left(\begin{array}{l}
x_{0} \\
y_{0}
\end{array}\right)
$$

with azimuth $\varphi$ and $s \in\left[s_{1}, s_{2}\right]$ and offset to the origin

$$
\left(\begin{array}{l}
x_{0} \\
y_{0}
\end{array}\right)=a \cdot\left(\begin{array}{c}
\sin (\varphi) \\
-\cos (\varphi)
\end{array}\right) \text {, }
$$

where $a \in \mathbb{R}$. 


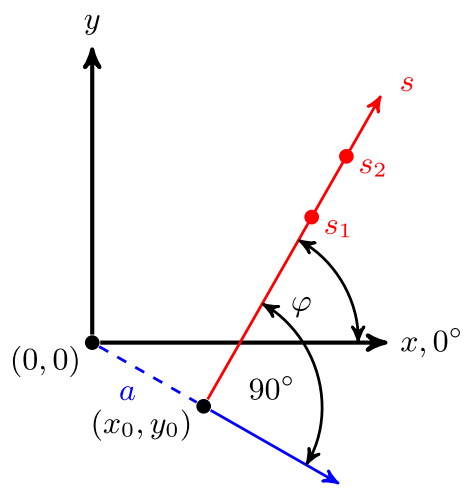

FIG. 2. Mathematical description of probe positioning. The probe orientation is given by $s$ with begin $s_{1}$ and end $s_{2}$. The offset of the probe from the machine center is indicated by the dashed line with symbol $a[1]$.

\section{B. Peak detection of probe measurement}

To determine the radial beam position at each turn, the radial profile from the wire probe needs to be analyzed. This is done with a robust and straightforward peak detection algorithm that searches for downward zero crossings in the smoothed first derivative with thresholds on the minimum peak value, area, and slope. The identified peaks of the measurements are indicated in Figs. 3 and 4 with red dots.

In order to estimate the error of the measurements, the reference measurement in Fig. 4 was compared to measurements with a lower and higher beam current with the same machine condition. The histogram of the changes in peak positions is shown in Fig. 5. As seen in the figure, a change in the beam current does not influence the peak positions significantly.

\section{Measurement of centered beam}

The beam is extracted from the PSI Ring cyclotron using an electrostatic extractor, the septum that is located in the gap between the last two turns. The standard production setup of the PSI Ring cyclotron makes use of a noncentered beam such that the beam gap for the septum is enlarged by the beam precession. In order to obtain a proper scan of the turn pattern with a long radial probe, the beam has first to be centered accurately enough that individual turns are well separated. Only then is it possible to accurately count the number of turns $[15,16]$.

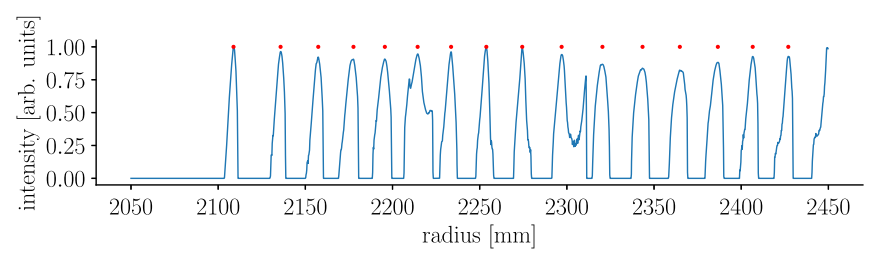

FIG. 3. Histogram of the probe RRI2 measurement. The intensity is normalized. The red dots mark detected peaks.
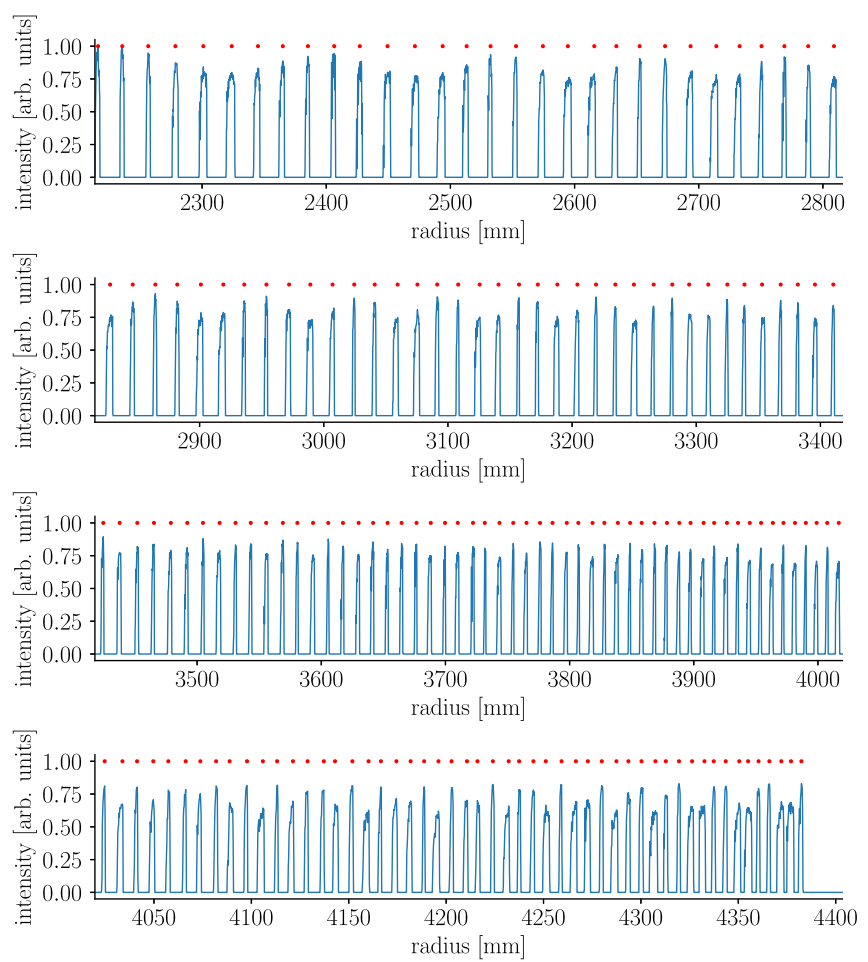

FIG. 4. Histogram of the probe RRL measurement. The intensity is normalized. The red dots mark detected peaks.

The beam centering of the PSI Ring cyclotron is determined by the beam energy, radius, and angle. The former is fixed by the extracted beam energy from injector 2, but the latter can be manipulated by the last two injection magnets AND1 and AND2 and the voltage of the electrostatic injection channel (EIC) (cf. Fig. 1). The centering of the beam is quantified by a numerical analysis of the data of a radial injection probe (RRI2). The radial positions $r_{n}$ of turn number $n$ can approximately be described by

$$
r_{n}=r_{0}+\left\langle\frac{d r}{d n}\right\rangle n+A \sin \left(2 \pi \nu_{r} n+\phi\right)
$$

where $\nu_{r}$ is the radial tune, $A$ is the betatron amplitude, and $\phi$ is the betatron phase. The radius gain per turn $\left\langle\frac{d r}{d n}\right\rangle$ can be

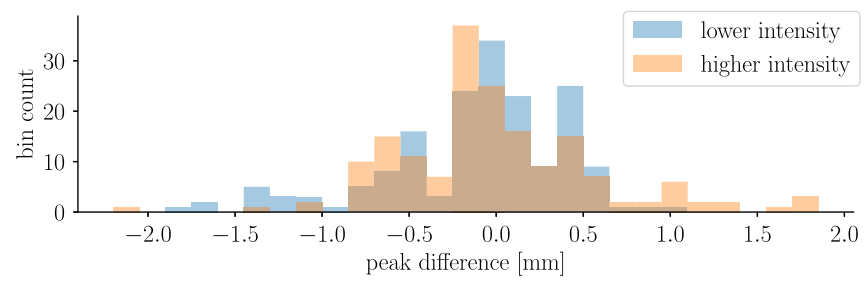

FIG. 5. Histogram (bin width $0.15 \mathrm{~mm}$ ) of the changes in the peak positions for the RRL probe compared to the reference measurement in Fig. 4 for a lower $(58 \mu \mathrm{A}, \mu=-0.1 \mathrm{~mm}, \sigma=0.5 \mathrm{~mm})$ and higher $(108 \mu \mathrm{A}, \mu=0.0 \mathrm{~mm}, \sigma=0.6 \mathrm{~mm})$ intensity. The mean absolute error (MAE) taking both intensities is $0.4 \mathrm{~mm}$. 


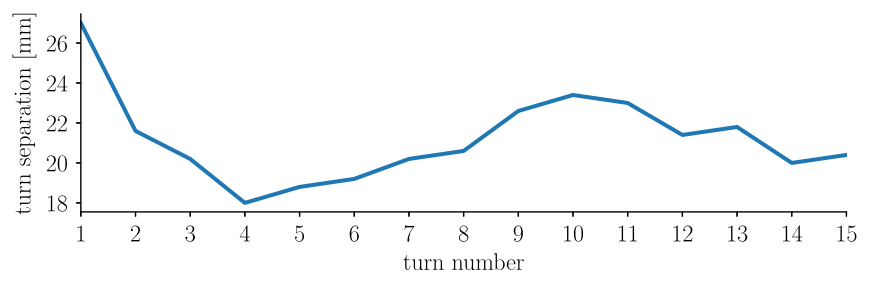

FIG. 6. Turn separation among subsequent orbits measured at the probe RRI2 (minimum $18.0 \mathrm{~mm}$ and maximum $27.0 \mathrm{~mm}$ ).

assumed to be approximately constant over a small range of turns where adjacent turns do not overlap if $2 A$ is smaller than the radius gain. For a centered beam, the currents of AND1 and AND2 have to be chosen such that $A \approx 0$. A straightforward method, used also at PSI, is to measure the two-dimensional maps $A\left(I_{1}, I_{2}\right)$ and $\phi\left(I_{1}, I_{2}\right)$, where $I_{1}$ is the current in AND1 and $I_{2}$ the current in AND2, respectively. Then $A$ and $\phi$ can be interpolated.

The probe measurements used in this paper are performed with a beam intensity of $88 \mu \mathrm{A}$. The beam profiles are given in Figs. 3 and 4. The corresponding turn separation, i.e., the distance between neighboring turns, at the probes is shown in Figs. 6 and 7. At injection, the turn separation is at maximum $27 \mathrm{~mm}$, which shrinks to $6.1 \mathrm{~mm}$ for the last 20 turns.

\section{Trim coils}

The PSI Ring cyclotron is equipped with 18 trim coils which allow compensation of field errors and manipulation and optimization of the beam phase and isochronism. The trim coils are referred to as TC1-TC18, from small to large radius. The trim coils TC6-TC14 are positioned only on top of the odd numbered sector magnets, i.e., SM1, SM3, etc. The other trim coils are on all magnets.

The trim coils allow one to change the field and to shift the beam phase. This can also alter the energy gain per turn. Furthermore, the trim coils enable, within certain limits, the manipulation of the tune diagram of the ring cyclotron and, thus, to either avoid resonances or change their position and influence on the beam.

As mentioned in the introduction, the lack of magnetic field data of the trim coils makes it currently impossible to model the fields accurately. Therefore, the simulation model developed in this paper is based on the average

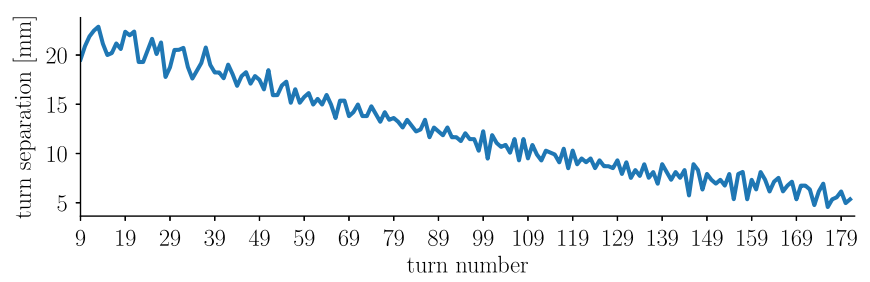

FIG. 7. Turn separation among subsequent orbits measured at the probe RRL (minimum $4.6 \mathrm{~mm}$ and maximum $22.9 \mathrm{~mm}$ ). field profiles obtained by measurements of the beam phase shifts as subsequently explained.

\section{Measurement fitting}

The presented trim coil model is based on measurements of $\Delta \sin (\varphi)$ [17] in Eq. (4) as depicted in Fig. 8 with beam phase $\varphi$. As stated in Ref. [18], the beam phase relates to $\Delta B_{k}$, the magnetic field change due to trim coil $k$, by

$$
\Delta B_{k} \sim-\frac{q B(r) V(r) r}{E(r) \gamma(r)[\gamma(r)+1]} \frac{d \sin (\varphi)}{d r}
$$

with radius $r$, magnetic field $B(r)$, energy gain $V(r)$, charge $q$, kinetic energy $E(r)$, and relativistic factor $\gamma(r)$. In the development of the trim coil model, the simplified relation

$$
\Delta B_{k} \sim-\frac{d \sin (\varphi)}{d r}
$$

was used instead. Since the neglected factor of Eq. (2) varies little over the radial range of a single trim coil, the negligence in Eq. (3) does not deteriorate the model. In order to obtain the magnetic field magnitude as an additional degree of freedom, the numerical model relies on normalized fields as discussed later. Each trim coil phase data point was approximated by a rational function, i.e.,

$$
[\Delta \sin (\varphi)](r) \approx \frac{f(r)}{g(r)}=\frac{\sum_{i=0}^{n} a_{i} r^{i}}{\sum_{j=0}^{m} b_{j} r^{j}}
$$

with $m, n \in \mathbb{N}_{0}$ and $m>n$. The coefficients were computed by a PYTHON script using the nonlinear least-squares method of SCIPY [19], where $n=2, m=4$ for trim coils TC2-TC15 and $n=4, m=5$ for TC1 and TC16-TC18, respectively. The fits of the data are shown in Fig. 9. The selection of the parameters $n$ and $m$ was done empirically trying to keep the polynomial degree small. As a result of

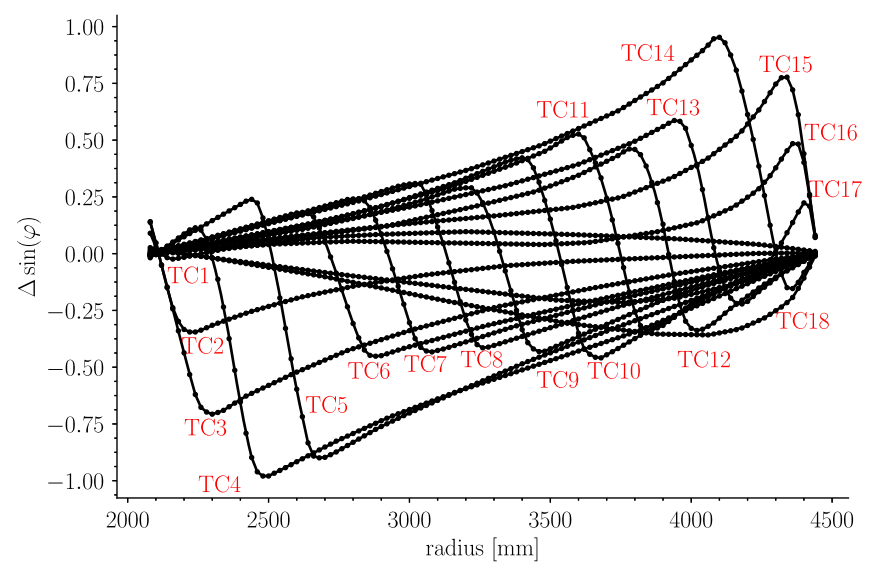

FIG. 8. Measurement of the beam phase $\varphi$ shift due to trim coils [17]. 

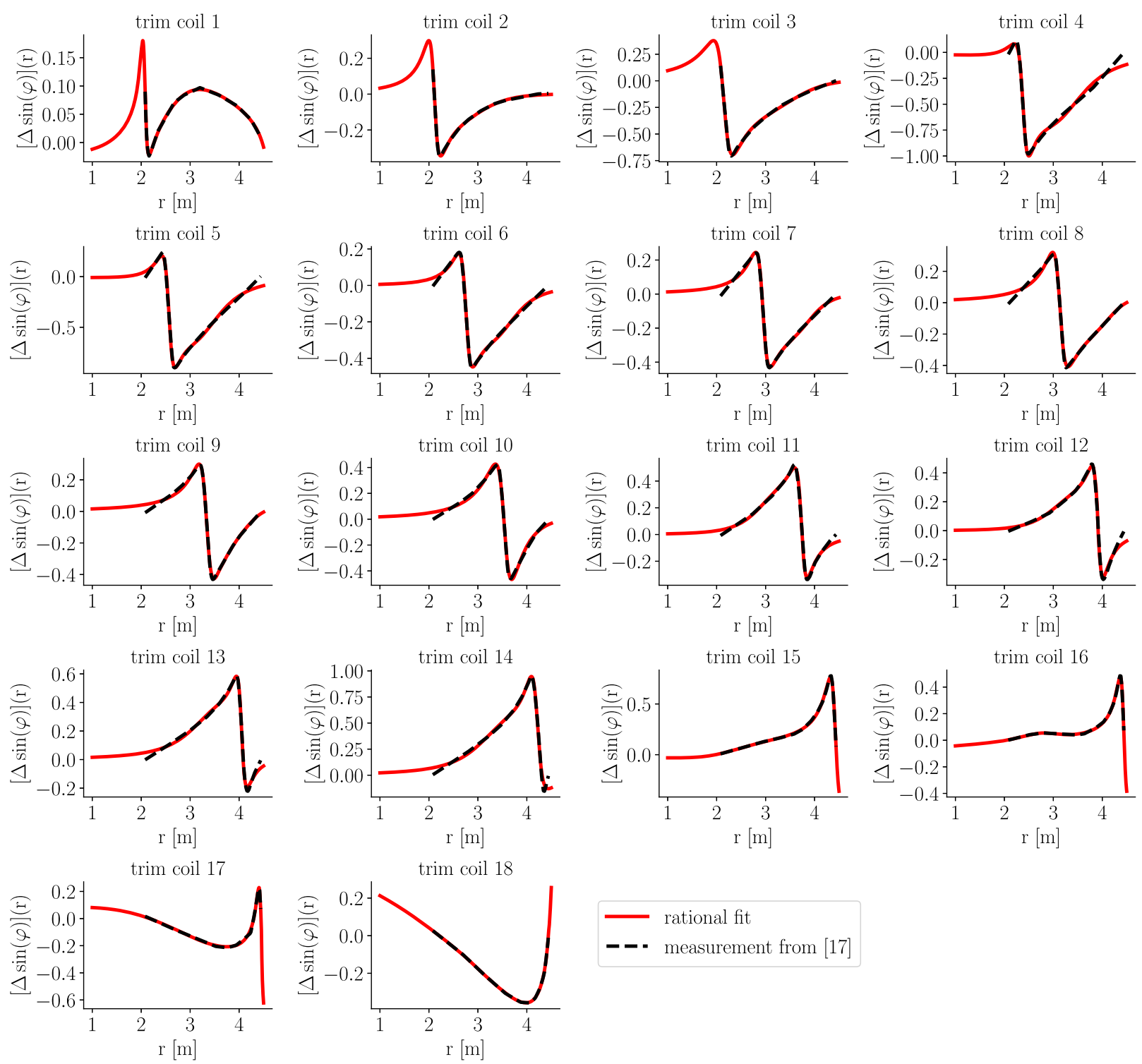

- rational fit

- - - measurement from $[17]$

FIG. 9. Fits of the $\Delta \sin (\varphi)$ measurements using rational functions. The change of the beam phase $\varphi$ is induced by the trim coil fields.

Eq. (4), the corresponding magnetic field is therefore simply given by

$$
B(r) \sim \frac{d}{d r} \frac{f(r)}{g(r)}=\frac{f(r) g^{\prime}(r)-f^{\prime}(r) g(r)}{g^{2}(r)},
$$

with $f^{\prime}(r) \equiv d f(r) / d r$. The normalized magnetic field and its derivative of each trim coil are depicted in Fig. 10.

\section{OPAL}

The open source library OPAL [13] is a parallel electrostatic particle-in-cell framework for large-scale particle accelerator simulations. In what follows, the OPALcycl flavor that is used for all simulations in this study is also referred as OPAL. The particles are evolved in time $t$ by either a fourth-order Runge-Kutta or a second-order leapfrog according to the collisionless Boltzmann (or Vlasov-Poisson) equation

$$
\frac{d f}{d t}=\frac{\partial f}{\partial t}+\mathbf{v} \cdot \nabla_{\mathbf{x}} f+\frac{q}{m_{0}}(\mathbf{E}+\mathbf{v} \times \mathbf{B}) \cdot \nabla_{\mathbf{v}} f=0,
$$

with charge $q$, mass $m_{0}$, and the six-dimensional particle density function $f(\mathbf{x}, \mathbf{v}, t)$ with $(\mathbf{x}, \mathbf{v}) \in \mathbb{R}^{3 \times 3}$. The electromagnetic fields $\mathbf{E} \equiv \mathbf{E}(\mathbf{x}, t)$ and $\mathbf{B} \equiv \mathbf{B}(\mathbf{x}, t)$ consist of a bunch internal and external contribution. The bunch selffield is obtained in the beam rest frame by either a FFT Poisson solver or a smoothed aggregation algebraic multigrid [20] solver that is able to handle arbitrary accelerator geometries.

The following subsections highlight three features of OPAL that were used as well as extended for the purpose of this study. 

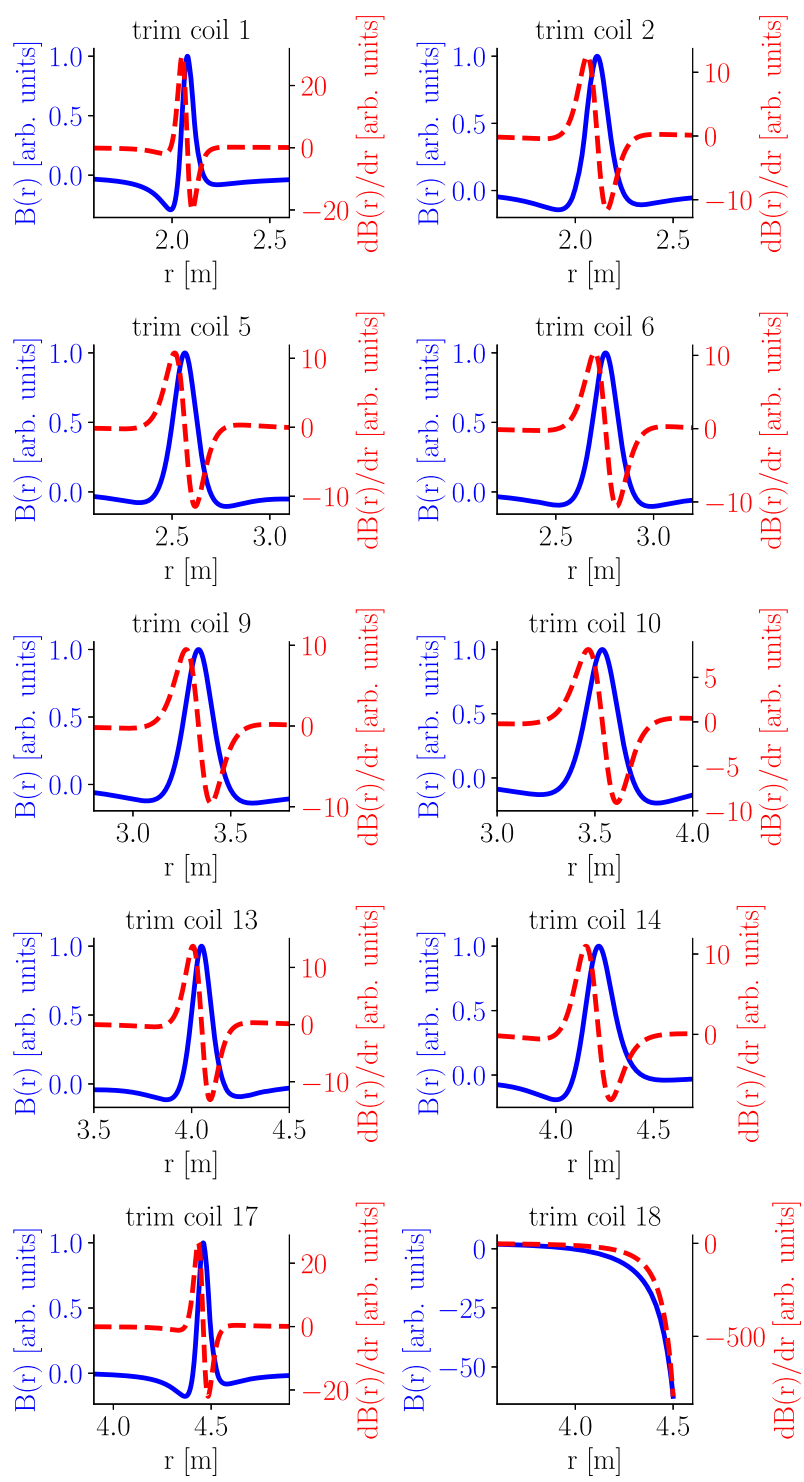
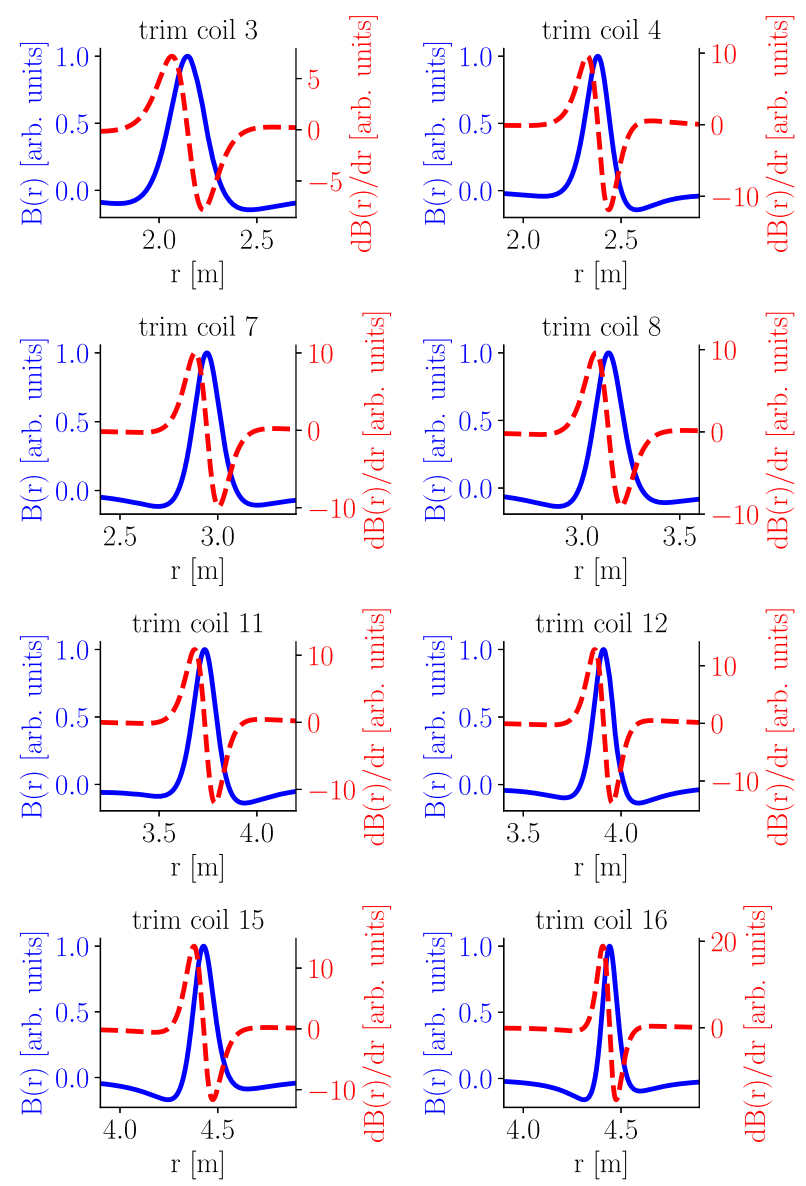

FIG. 10. Magnetic fields and their radial derivative of the trim coils.

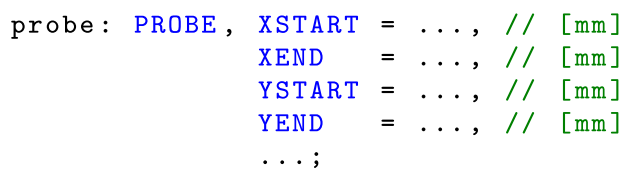

FIG. 11. OPAL input command for probe elements.

\section{A. Probe element and peak detection}

In the cyclotron flavor of OPAL, the probe is a special element placed on the midplane in Cartesian coordinates to record particles in simulations. The origin is the machine center; therefore, the positioning according to Eq. (1) with Fig. 2 is directly applicable. The OPAL syntax is shown in Fig. 11. Since OPAL has fixed time steps, particle position recording at the probe is done by a linear extrapolation of the particle direction from the closest tracking point towards the probe axis. In order to compare the measurement and simulation, the original description in OPAL was extended to write a particle histogram and a file collecting the peak locations. In single particle tracking, the peak and, thus, turn detection are trivial. The localization of a turn in a multiparticle simulation is achieved by summing up all radii where particles hit the probe. The mean is then determined as the orbit radius.

\section{B. Multiobjective optimization}

Since release version 2.0.0, OPAL is equipped with a multiobjective genetic algorithm NSGA-II implementation [21]. The new OPAL feature was already applied in Ref. [22]. Initially, a population of $n$ randomly spawned individuals within a predefined hyperspace of design 
variables is created, after which a new set of individuals for the next population within the same bounds is selected by mixing the current $k$-fittest individuals based on a crossover pattern as well as a gene mutation algorithm. The fitness of an individual is determined by the evaluation of userdefined functions on the objectives that in this paper are the peak differences between the measurement and simulation. In this regard, the framework was extended to be able to read peak files and to evaluate the $l_{\infty}$-error and $l_{2}$-error norms on the parsed peak data. Furthermore, an expression to check the number of turns in simulations was added that served as an individual constraint. One of the main benefits of MOGA is its ability to search in parallel, as it can evaluate all individuals of a single generation at the same time.

\section{New trim coil model}

The existing trim coil model in OPAL [3] was especially designed to fit the shape of TC15 of the PSI Ring cyclotron, because its interest focused on the turns close to extraction. Furthermore, the field was contributed not only local to the sector magnets but continuously smeared out on $360^{\circ}$. The new model uses a more general description by rational functions as described in Sec. II D 1. This representation of the field allows a simple analytical differentiation to obtain the necessary derivative for the magnetic field interpolation to the position of each particle. That way, the model is not restricted to the specific shape of TC15 in Ref. [3].

The new trim coil model does not support an azimuthally limited field definition. However, the trim coil fields are restricted to the sector magnets by a user-defined threshold that is the lower limit to apply the additional fields. The implementation of the trim coils assumes normalized polynomial coefficients such that the maximum value of the field is 1.0; thus, the maximum field strength $B_{\max }$ is an auxiliary tuning parameter, i.e.,

$$
\mathrm{TC}(r)=B_{\max } \frac{\sum_{i=0}^{n} a_{i} r^{i}}{\sum_{j=0}^{m} b_{j} r^{j}}
$$

with $n, m \in \mathbb{N}_{0} \wedge r \in\left[r_{\min }, r_{\max }\right]$. Second, the trim coil field is restricted in the radial direction by two extra parameters $r_{\min }$ and $r_{\max }$ to allow more flexibility. Nevertheless, the bounds have to be selected carefully to avoid a discontinuity in the magnetic field. In the azimuthal direction, the implementation uses a linear decaying field to prevent the previously mentioned issue. Since the functions $f(r)$ and $g(r)$ in Eq. (4) are polynomials in radius $r$, the derivative Eq. (5) is a rational function again. The model can therefore accept either the phase or magnetic field as input.

A template of a trim coil definition in an OPAL input file is given in Fig. 12. The parameter TYPE specifies if the polynomial represents the phase PSI-PHASE or the magnetic field PSI-BFIELD. In order to be applied, the trim coil

$\begin{aligned} & \text { tc1: TRIMCOIL, TYPE }=" \text { PSI-PHASE" } \\ & \text { RMIN }=\ldots, / / \text { inner radius }[\mathrm{mm}] \\ & \text { RMAX }=\ldots, / / \text { outer radius }[\mathrm{mm}] \\ & \text { BMAX }=\ldots, / / \text { B-field peak }[\mathrm{T}] \\ & \text { COEFNUM }=\{\mathrm{a} 0, \mathrm{a} 1, \mathrm{a} 2, \ldots\}, \\ & \text { COEFDENOM }=\{\mathrm{b} 0, \mathrm{~b} 1, \mathrm{~b} 2, \ldots\} ;\end{aligned}$

FIG. 12. OPAL input command for trim coils.

\begin{tabular}{ll}
\hline & $/ /$ lower limit of $\mathrm{TC}$ input $[\mathrm{T}]$ \\
Ring: CYCLOTRON, & TRIMCOILTHRESHOLD $=\ldots$, \\
& TRIMCOIL $=\{\mathrm{tc1}, \mathrm{tc2}, \mathrm{tc} 3, \ldots\}$ \\
& $\ldots$ \\
& $;$
\end{tabular}

FIG. 13. Additional trim coil input arguments for the cyclotron element definition in OPAL.

elements have to be appended to a list in the cyclotron command as depicted in Fig. 13.

\section{TURN PATTERN MATCHING}

A measure for the quality of the pattern matching is the maximal peak difference between measurement $m$ and simulation $s$, i.e.,

$$
\min \max _{i=1 \ldots N}\left|r_{i}^{m}-r_{i}^{s}\right|
$$

where $N$ is the number of turns and $r_{i}^{m}$ and $r_{i}^{s}$ are the $i$ th turn radii.

An iterative process to get a model that is in good agreement with measurements applied multiobjective optimization and local search. Furthermore, the input parameter space between different optimizations was flexible; i.e., design variables (DVARs) were added and removed. The selection of the design variables is described in detail in the subsequent section.

Because of the decrease of the turn separation as discussed in Sec. II C and the increase of the circumference of the machine, the choice of the number of steps per turn in a simulation needs to be chosen carefully to obtain reliable results. Furthermore, the extrapolation method that is used to get the point where the particle hits the probe depends also on this time discretization. After a comparison between different numbers of steps per turn and a reference simulation with 23040 integration steps per turn (cf. Figs. 15 and 16), the optimal number of steps per turn for the fourth-order Runge-Kutta integrator with respect to accuracy and run time turned out to be 2880 . It compares to the reference simulation with a maximum absolute error of $1.5 \mathrm{~mm}$, MAE $0.6 \mathrm{~mm}$, and mean squared error (MSE) $0.4 \mathrm{~mm}^{2}$. The reference simulation is selected based on the observation that the turn radii differ only in the order of $\mathcal{O}(0.2) \mathrm{mm}$ at the probes compared to 11520 steps 


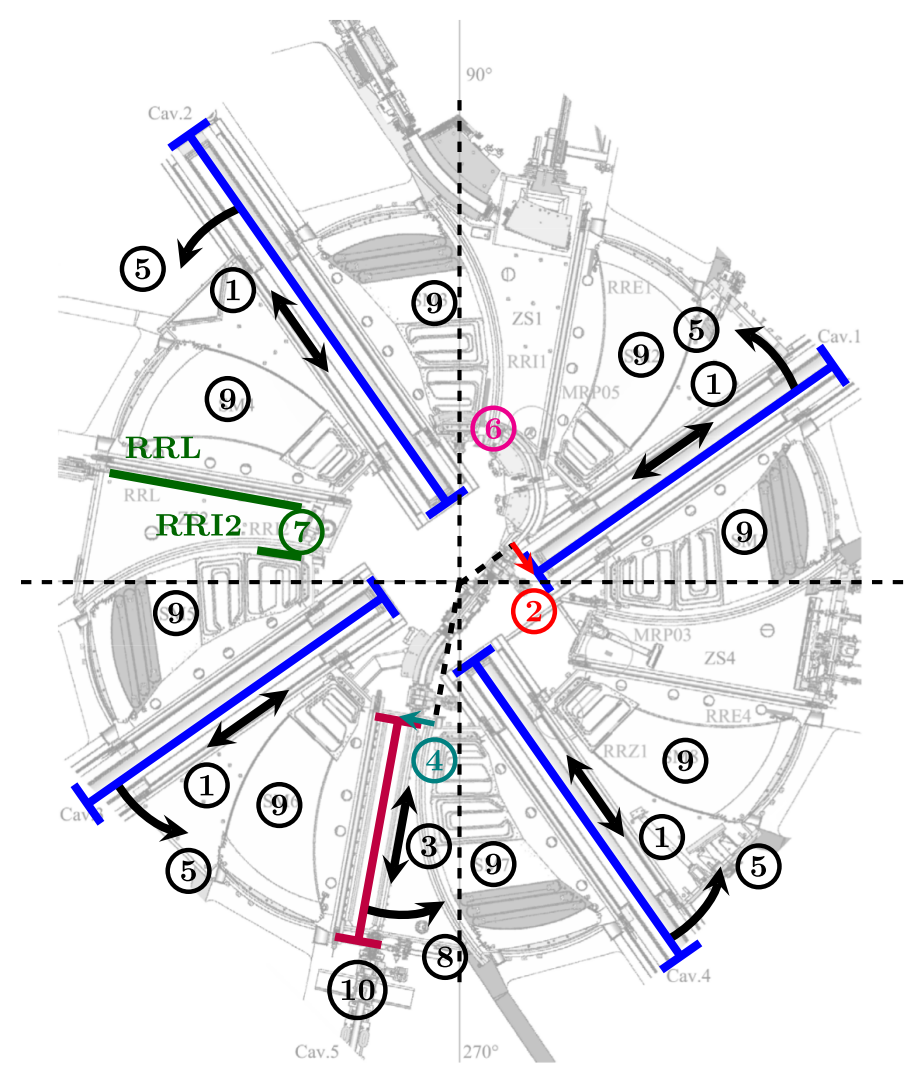

FIG. 14. Design variables in context of the PSI Ring cyclotron. In Table V is a description of each variable. Legend: (1), rmainshift1rmainshift4 and vmaincav1—vmaincav4; (2), pdismain1—pdismain4; (3), rftshift; (4), pdisft; (5), phimain1—phimain4; (6), benergy, prinit, phiinit, and rinit; (7), rrla, rrlphi, rrlshift, rri2a, rri2phi, and rri2shift; (8), phift; (9, tc01mb-tc16mb; (10, phirfft and vftcav.

per turn. A significant improvement is achieved only with an integrator of higher order.

\section{A. Design variable selection}

As previously mentioned, the selection of the design variables was not obvious at first. Initially, the beam injection parameters and the peak magnetic field of the first trim coils were considered in order to match the first turns at injection. However, the idea of matching basically turn by turn starting at injection failed soon, since the turn pattern difference between the measurement and simulation started to diverge at later turns due to the wrong energy gain per turn. As a consequence, the rf cavity parameters together with a constraint on the number of completed turns were added to guide the optimization towards solutions with the right number of turns. For rf cavities, their voltages and positions were varied where a position encompasses the angle, radial position, and displacement from the global center. For the flattop cavity, also the phase angle was added as a parameter. The angle between RRI2 and RRL and their radial position were varied in order to smooth the transition between the probes.

Since TC18 is turned off, it was not added as design variable. Also, TC17 that influences the last few turns was discarded, since the extraction channel is not simulated. However, these turns are still corrected in the simulation by TC16.

The final list of 48 DVARs is given in Table V of the Appendix. As a further clarification, they are also depicted in the drawing of the cyclotron in Fig. 14. The angle between the probes RRI2 and RRL as indicated by (7) in the plot is adjusted using the variables $a$ and $\varphi$ of Eq. (1) while keeping the length of each probe $s \in\left[s_{0}+t, s_{1}+t\right]$ with $t \in \mathbb{R}$ fixed.

\section{B. Model simplifications}

Beside numerical approximations on the design of the new trim coil model, the large number of DVARs (i.e., 48) and objectives (i.e., 182 turns) required further simplifications on the optimization approach as explained in the following.

\section{Aggregation of turns}

In the case of the PSI Ring cyclotron, the number of objectives (i.e., turns) is 182 . In order to reduce this space, multiple turns were clustered to single objectives $\sigma_{[l, u]}$ with turns in the range $[l, u] \in[1,182]$ by either the $l_{2}$-error norm 


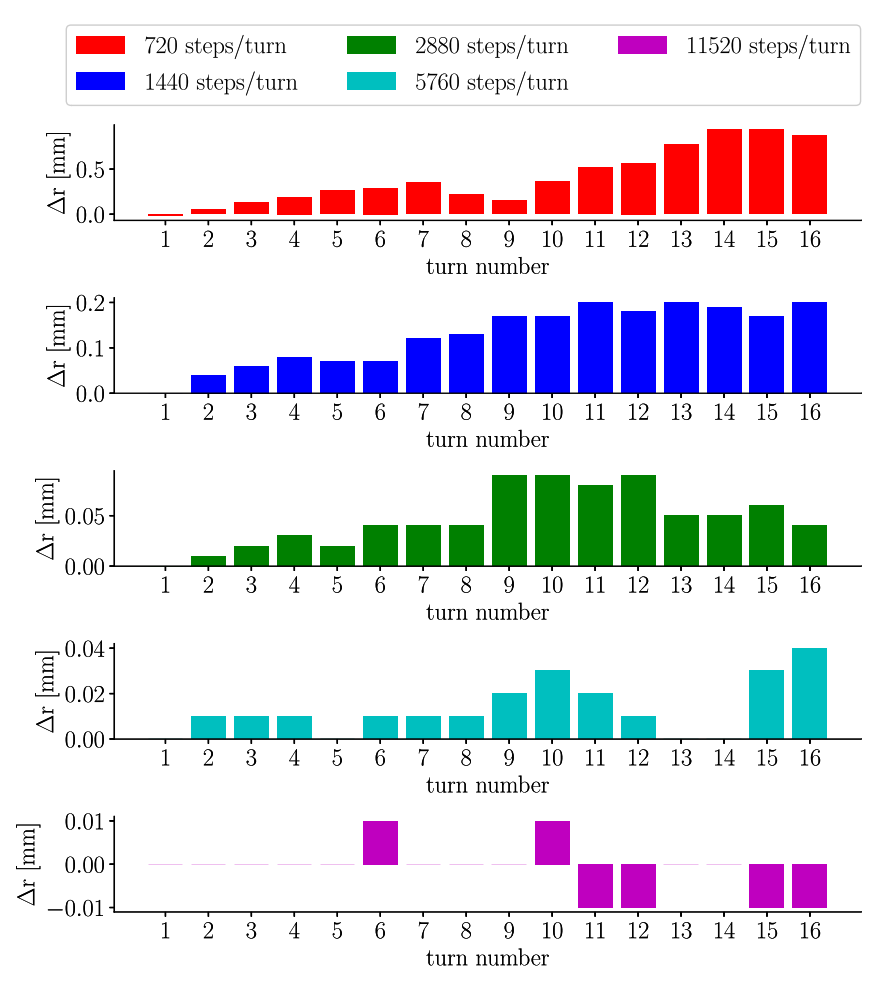

FIG. 15. Difference of the turn radius $\Delta r$ at probe RRI2 between simulations due to the number of integration steps per turn. The reference simulation uses 23040 steps per turn. The input parameters of the simulation are optimized for 2880 steps per turn.
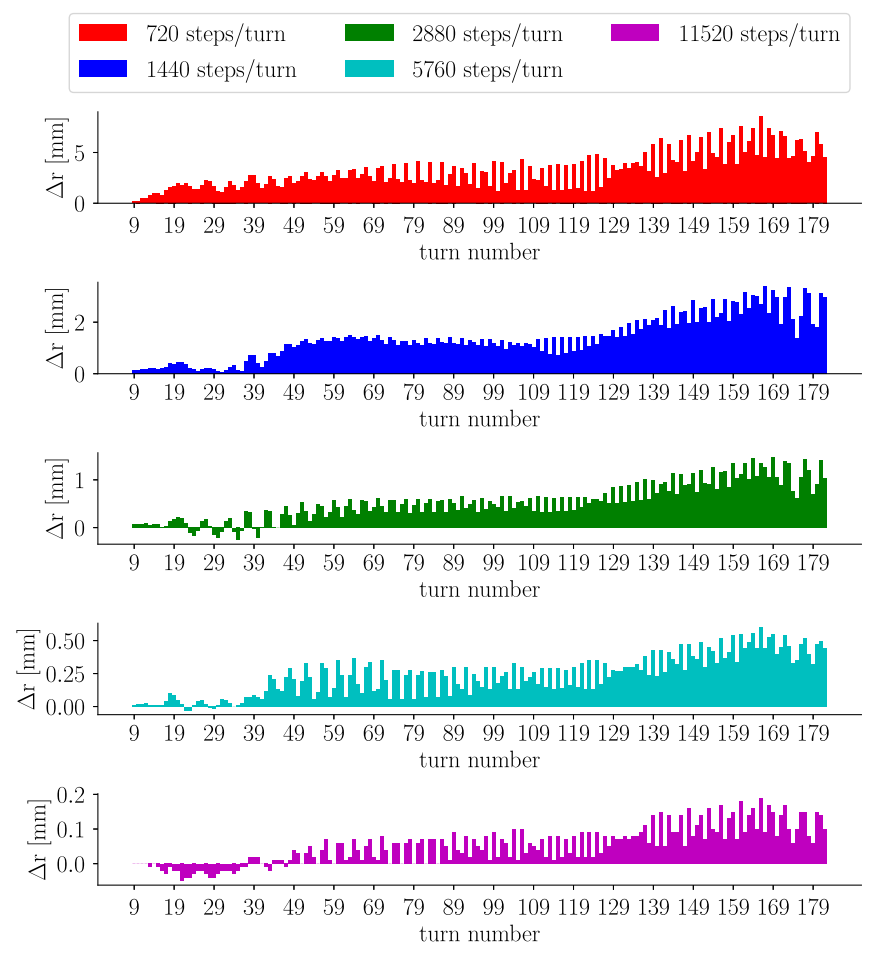

FIG. 16. Difference of the turn radius $\Delta r$ at probe RRL between simulations due to the number of integration steps per turn. The reference simulation uses 23040 steps per turn. The input parameters of the simulation are optimized for 2880 steps per turn.

$$
\sigma_{[l, u]}=\frac{1}{N} \sqrt{\sum_{i=l}^{u}\left(r_{i}^{m}-r_{i}^{s}\right)^{2}}
$$

with $N=u-l+1$ the number of aggregated turns or the $l_{\infty}$-error norm

$$
\sigma_{[l, u]}=\max _{i=l \ldots u}\left|r_{i}^{m}-r_{i}^{s}\right|
$$

where $r_{i}^{m}$ and $r_{i}^{s}$ are the $i$ th turn radii of the measurement and simulation, respectively. The $l_{\infty}$ norm suits our definition of the measure for the pattern matching quality, i.e., Eq. (6).

\section{Reduction of trim coil support}

Because of the field overlap of neighboring trim coils, a valid assumption is the partial cancellation of the field tails. That is why the model uses a reduced radial support. Only trim coil TC1 uses the full range on the lower half.

\section{Location of trim coils}

In order to limit the trim coil field in the azimuthal direction, the user provides a lower bound of the magnetic field by the attribute TRIMCOILTHRESHOLD (cf. Fig. 13), above which the trim coil field is applied. This is a limitation of the new model, since the real machine provides the field of all trim coils only on specific sector magnets (cf. Sec. II D).

\section{Single particle tracking}

The radial profiles are measured using a low-intensity beam, i.e., $88 \mu \mathrm{A}$. The negligence of space charge in order to lower the time to the solution of a single simulation is therefore a reasonable assumption. A further simplification to single particle tracking is motivated by the observation that peaks are detected at the centroid of the beam (cf. Figs. 3 and 4). This reduces the time to model the full machine to approximately $2 \mathrm{~s}$ on a single core.

\section{Multiobjective optimization}

The dimension of the design variable space required a rather large number of individuals per generation in order to sample the space sufficiently. All optimizations were performed on Piz Daint [23], a supercomputer of the Swiss National Supercomputing Centre (CSCS). Because of its hardware architecture, where a node is equipped with two Intel Xeon E5-2695 v4 @ $2.1 \mathrm{GHz}(2 \times 18$ cores, 64/128 GB RAM) processors, a total number of 8062 individuals was selected in which two cores of the 224 nodes $(36 \times 224-2=8062)$ were reserved for individual postprocessing and bookkeeping. Since a single objective according to Sec. IV B 1 did not perform well, the turns were grouped into a total of finally six objectives. A reason might be local optima from which MOGAs are more likely 


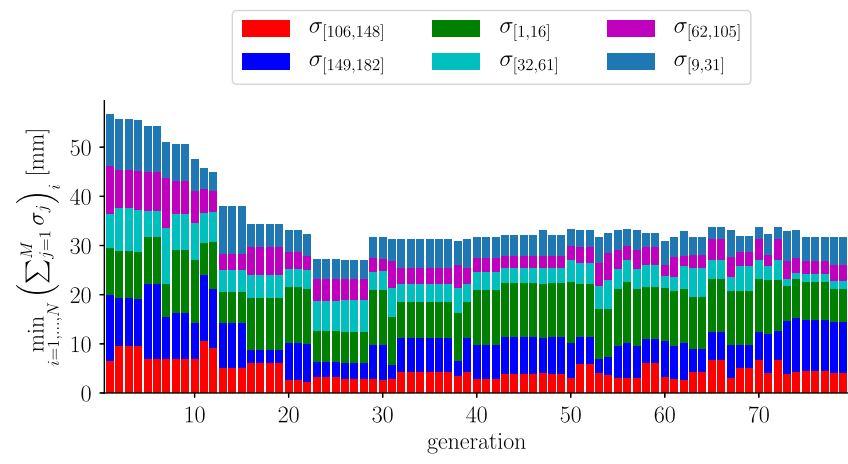

FIG. 17. Evolution of the best individual during the multiobjective optimization. The best individual of a generation is identified by the smallest sum of objectives $\sigma_{j}$ with $j \in[1, \ldots, M]$ and $M$ the number of objectives. The best individual was contained first in generation 26. The minimization is over all $N=8062$ individuals per generation. The label $\sigma_{[l, u]}$ indicates an objective for the turns in the range $[l, u]$.

to escape as discussed in Ref. [24]. While RRI2 was kept as a single objective, RRL was split into five objectives where each had approximately the same amount of turns and was influenced by a single trim coil only.

The optimization consisted of several independent runs with initially large bounds for each design variable. These bounds were narrowed according to the best individual. A best individual per generation is defined as the smallest sum of all $M$ objectives $\sigma_{j}$, i.e.,

$$
\min _{i=1, \ldots, N}\left(\sum_{j=1}^{M} \sigma_{j}\right)_{i}
$$

We show the evolution of only the last optimization in Fig. 17 that was stopped after 79 generations, since after 26 generations no significant error reduction was observed.

The objective values of the best individual over all generations are summarized in Table I. According to Fig. 6, the smallest turn separation at RRI2 is $18 \mathrm{~mm} \gg$ $\sigma_{[1,16]}=6.4 \mathrm{~mm}$, where the symbol $\sigma_{[l, u]}$ indicates a single objective for the turns $l$ to $u$. Therefore, the deviation to the measurement is less than half a turn for the maximum

TABLE I. Result of the best individual obtained by optimization using the $l_{\infty}$-error norm for each objective. The label $\sigma_{[l, u]}$ indicates an objective for the turns in the range $[l, u]$.

\begin{tabular}{lcl}
\hline \hline Objective $\sigma_{[l, u]}$ & $l_{\infty}$ error $(\mathrm{mm})$ & Probe \\
\hline$\sigma_{[1,16]}$ & 6.4 & RRI2 \\
$\sigma_{[9,31]}$ & 3.8 & RRL \\
$\sigma_{[32,61]}$ & 6.3 & RRL \\
$\sigma_{[62,105]}$ & 4.4 & RRL \\
$\sigma_{[106,148]}$ & 2.9 & RRL \\
$\sigma_{[149,182]}$ & 3.3 & RRL \\
\hline \hline
\end{tabular}

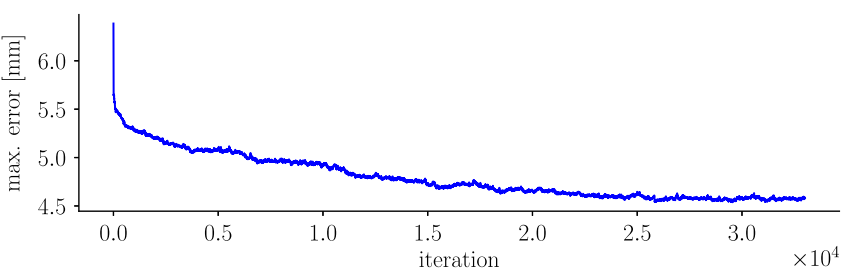

FIG. 18. Evolution of the $l_{\infty}$ error between the measurement and simulation during the local search with the best individual obtained by the MOGA as the starting point.

absolute error. In the case of RRL, the difference is also always below the turn separation (cf. Fig. 7).

\section{Local search}

While the genetic algorithm can, in principle, search a large variable space effectively, it was not able to find a nearby better solution in a reasonable amount of time. We suspect this is due to the high sensitivity of the design variables near the optimum, the heuristics of genetic algorithms, and the large dimensionality of the design variable space. Therefore, once the best individual from the genetic algorithm was selected, a local search around this individual was done to find the optimum. The chosen local search involved changing a single parameter value iteratively. This approach reduced the turn pattern error significantly.

Defining a good metric for the search was crucial, since the iterative search is likely to stop in a local optimum. To avoid local optima, several norms were used simultaneously, namely, the maximal error $\left(l_{\infty}\right.$ error), the second largest error, the third largest error, and a weighted $l_{2}$ error where the RRI2 turns were weighted equally to the RRL turns. A parameter was allowed to change when there was an improvement in any of the norms while not worsening the other norms significantly $\left(0.01 \mathrm{~mm}\right.$ for the $l_{\infty}$ error $)$. This is equivalent to a multiobjective optimization. In Fig. 18, the $l_{\infty}$ error is shown during the iterative search. It can be seen that there was a significant improvement in the beginning, reducing the error from more than $6 \mathrm{~mm}$ to less than $5 \mathrm{~mm}$. It can also be seen that the error occasionally increases, which avoids the local optima. Once no

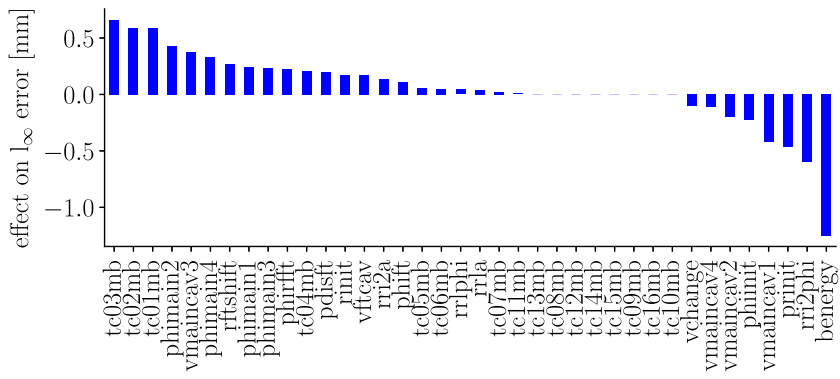

FIG. 19. Total effect during the local search on the $l_{\infty}$ error per design variable. 


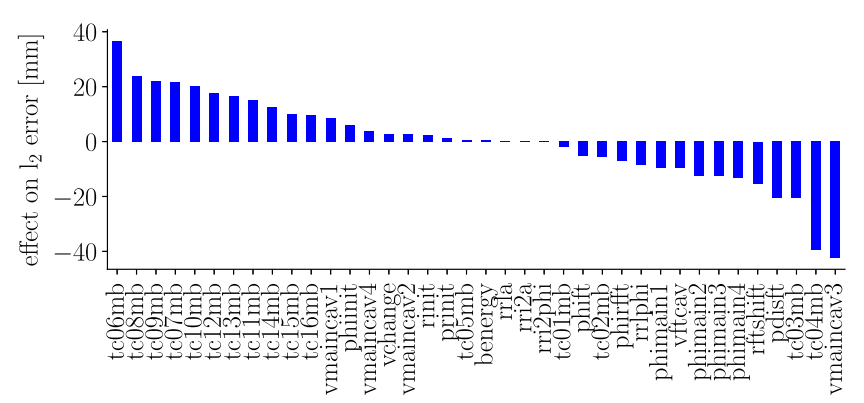

FIG. 20. Total effect during the local search on the $l_{2}$ error per design variable.

improvement in the $l_{\infty}$ error over 30000 iterations was observed, the local search was stopped.

In Figs. 19 and 20, the effect on the $l_{\infty}$ error and $l_{2}$ error per design variable is shown. It can be seen that, as expected, the trim coils generally improve the $l_{2}$ error, while the first trim coils and rf improve the $l_{\infty}$ error. The explanation for the latter is that the largest mismatch during the scan was often in one of the first turns.

\section{DISCUSSION}

The starting point of the local search was the best individual of the MOGA as explained in Sec. IV D. This additional step could reduce the error spread and maximum absolute error (at turn 2) compared to the measurement (cf. Table II). The error of the turn radius of both methods is shown in Figs. 21 and 22.

The result of the single particle local search is verified with two multiparticle tracking simulations at $88 \mu \mathrm{A}$ having 360000 macroparticles and space charge (i.e., FFT Poisson solver) switched either on or off. The multiparticle tracking (no space charge) changes the error compared to the measurement only slightly. The maximum absolute error is increased by $0.1 \mathrm{~mm}$ in comparison to the single particle simulation. The MAE and mean squared error rise only by +0.1 and $+0.2 \mathrm{~mm}^{2}$, respectively.

A multiparticle tracking simulation with space charge does not change the turn pattern perceptibly (cf. Table III). The $l_{\infty}$ error between both multiparticle simulations differs by $0.05 \mathrm{~mm}$ and MAE $0.00 \mathrm{~mm}$. These observations confirm the model assumptions to neglect space charge and to use a single particle only in order to match the turn pattern.

TABLE II. Maximum absolute error ( $l_{\infty}$ norm), MAE, and MSE of the best individual of the optimizer and local search compared to the measurement. In both cases, the maximum error is at turn 2 .

\begin{tabular}{lccc}
\hline \hline Method & $l_{\infty}$ norm $(\mathrm{mm})$ & MAE $(\mathrm{mm})$ & MSE $\left(\mathrm{mm}^{2}\right)$ \\
\hline Optimizer & 6.4 & 2.0 & 6.3 \\
Local search & 4.5 & 1.4 & 3.4 \\
\hline \hline
\end{tabular}

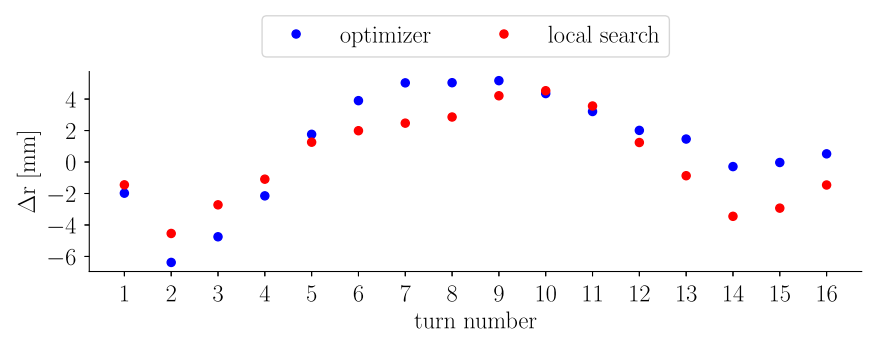

FIG. 21. Error of the turn radius at RRI2 between the measurement and simulation of the best individual obtained by multiobjective optimization and local search.

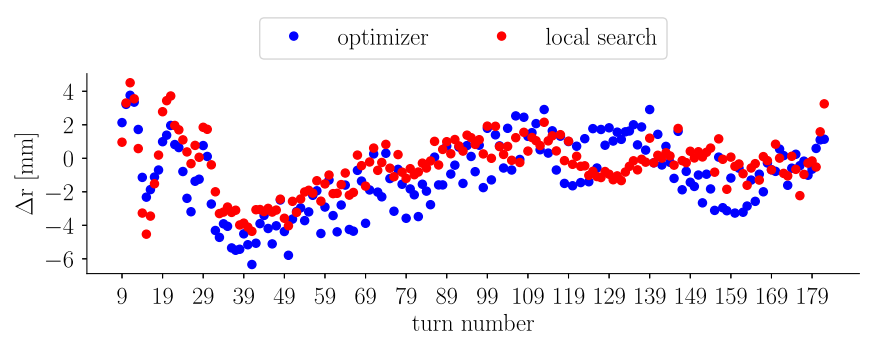

FIG. 22. Error of the turn radius at RRL between the measurement and simulation of the best individual obtained by multiobjective optimization and local search.

An estimation of the error due to the measurement and model simplifications is given in Table IV. The systematic error is $3.9 \mathrm{~mm}$, which is comparable to $4.5 \mathrm{~mm}$ of the local search (cf. Table II). The MAE also differs only by $0.3 \mathrm{~mm}$. The difference of the MSE is, however, $2.5 \mathrm{~mm}$.

TABLE III. Maximum absolute error $\left(l_{\infty}\right.$ norm), MAE, and MSE of the measurement or multiparticle tracking simulation including space charge to the multiparticle tracking simulation neglecting space charge.

\begin{tabular}{lccc}
\hline \hline Comparison to & $l_{\infty}$ norm $(\mathrm{mm})$ & MAE $(\mathrm{mm})$ & $\operatorname{MSE}\left(\mathrm{mm}^{2}\right)$ \\
\hline Measurement & 4.6 & 1.5 & 3.6 \\
Space charge & 0.1 & 0.0 & 0.0 \\
\hline \hline
\end{tabular}

TABLE IV. Estimation of the lower bound of the error due to model simplifications and measurement inaccuracies.

\begin{tabular}{lccc}
\hline \hline Error source & $\begin{array}{c}l_{\infty} \text { norm } \\
(\mathrm{mm})\end{array}$ & $\begin{array}{c}\text { MAE } \\
(\mathrm{mm})\end{array}$ & $\begin{array}{c}\text { MSE } \\
\left(\mathrm{mm}^{2}\right)\end{array}$ \\
\hline $\begin{array}{l}\text { Measurement (cf. Fig. 5) } \\
\text { Multiparticle }\end{array}$ & 2.2 & 0.4 & 0.3 \\
$\quad$ (no space charge) & 0.1 & 0.1 & 0.2 \\
$\begin{array}{l}\text { Space charge effect } \\
\text { Step size (cf. Sec. IV) }\end{array}$ & 0.1 & 0.0 & 0.0 \\
Sum & 1.5 & 0.6 & 0.4 \\
\hline \hline
\end{tabular}




\section{CONCLUSION}

A realistic simulation of existing cyclotrons heavily depends on measured data and the accurate parameter specification of the machine. Furthermore, the precision of the numerical models of devices such as rf cavities and radial probes as well as the time discretization have an impact on the result. While the latter is improved by a higher resolution at the expense of longer time to solutions of the simulation or a more accurate time integrator, numerical models of devices are only enhanced by better methods.

A new, more realistic trim coil model in the beam dynamics code OPAL that supersedes the model developed in Ref. [3] was presented. The model uses rational functions to describe the shape of the trim coil field in the radial direction. Although the model was applied to the PSI Ring cyclotron, it is applicable to any circular type of machine.

Thanks to the flexibility of the model, it could be used to match the turn pattern of the simulation with measurements of a centered beam in the PSI Ring cyclotron. In order to match all 182 turns, a multiobjective optimization was applied with a parameter space including 16 trim coils and 32 other design variables, such as beam injection energy, rf cavity voltages, and various element positions. The full list of design variables is given in Table V. This process was complemented with a local search starting from the best individual of the MOGA. That way, the absolute error between the simulation and measurement could be reduced to at most $4.5 \mathrm{~mm}$. Despite several simplifications on the optimization procedure, multiparticle tracking without space charge verified the matching of the single particle tracking. Nevertheless, the numerical model can be improved further; especially, the azimuthal location of the trim coil field could be enhanced and, if possible, a three-dimensional representation is aimed. In addition, future work will include the matching of a noncentered beam and the beam profile.

The proposed approach of multiobjective optimization is unique to this kind of problem and might be used as a guideline for future projects. One of them is the DAE $\delta$ ALUS project $[25,26]$, a proposed search for CP violation in the neutrino sector. The DAE $\delta$ ALUS Superconducting Ring Cyclotron shares many similarities with the PSI Ring cyclotron. Future design studies will benefit greatly from the newly developed methods that were presented here.

\section{ACKNOWLEDGMENTS}

The authors thank W. Joho, M. Humbel, R. Dölling, and H. Zhang for their helpful discussions about trim coils and probe measurements. The contribution of D. Winklehner regarding the DAE $\delta$ ALUS is gratefully acknowledged as well. Furthermore, we appreciate the expertise of
M. Kranjčevic regarding the multiobjective optimization using evolutionary algorithms. All optimizations were performed using the HPC resources provided by the Swiss National Supercomputing Centre (CSCS). This project is funded by the Swiss National Science Foundation (SNSF) under Contract No. 200021_159936.

\section{APPENDIX: DESIGN VARIABLES}

This section lists all design variables applied in the multiobjective optimization and local search in Table V. A visualization in context of the PSI Ring cyclotron is given in Fig. 14.

TABLE V. Design variable abbreviations and their meaning.

\begin{tabular}{|c|c|c|}
\hline Design variable & Unit & Meaning \\
\hline benergy & $\mathrm{GeV}$ & Injection beam energy \\
\hline pdisft & $\mathrm{mm}$ & $\begin{array}{l}\text { Displacement of flattop's } \\
\text { axis from global center }\end{array}$ \\
\hline pdismain1-pdismain4 & $\mathrm{mm}$ & $\begin{array}{l}\text { Displacement of main } \\
\text { cavity's axis from global } \\
\text { center }\end{array}$ \\
\hline phift & $\operatorname{deg}$ & $\begin{array}{l}\text { Flattop cavity angle with } \\
\text { respect to global } \\
\text { coordinate system }\end{array}$ \\
\hline phiinit & deg & Injection angle of beam \\
\hline phimain1-phimain4 & deg & $\begin{array}{l}\text { Main cavity's angle with } \\
\text { respect to the center line } \\
\text { of sector magnet } 1\end{array}$ \\
\hline phirfft & deg & Phase of flattop \\
\hline prinit & $\beta \gamma$ & Injection radial momentum \\
\hline rftshift & $\mathrm{mm}$ & $\begin{array}{l}\text { Flattop cavity displacement } \\
\text { in radial direction }\end{array}$ \\
\hline rinit & $\mathrm{mm}$ & $\begin{array}{l}\text { Injection radius with respect } \\
\text { to the global coordinate } \\
\text { system }\end{array}$ \\
\hline rmainshift1-rmainshift4 & $\mathrm{mm}$ & $\begin{array}{l}\text { Main rf cavity displacement } \\
\text { in radial direction }\end{array}$ \\
\hline rri2a & $\mathrm{mm}$ & $a$ of Eq. (1) for RRI2 \\
\hline rri2phi & $\operatorname{deg}$ & $\varphi$ of Eq. (1) for RRI2 \\
\hline rri2shift & $\mathrm{mm}$ & $\begin{array}{l}\text { Start position of RRI2 } \\
\text { in radial direction } \\
(>0 \text { : outwards })\end{array}$ \\
\hline rrla & $\mathrm{mm}$ & $a$ of Eq. (1) for RRL \\
\hline rrlphi & $\operatorname{deg}$ & $\varphi$ of Eq. (1) for RRL \\
\hline rrlshift & $\mathrm{mm}$ & $\begin{array}{l}\text { Start position of RRL in } \\
\text { radial direction } \\
(>0 \text { : outwards })\end{array}$ \\
\hline $\mathrm{tc} 01 \mathrm{mb}-\mathrm{tc} 16 \mathrm{mb}$ & $\mathrm{T}$ & $\begin{array}{l}\text { Trim coil maximum } \\
\text { magnetic field }\end{array}$ \\
\hline vchange & MV & $\begin{array}{l}\text { Extra rf voltage change of } \\
\text { main cavities (in total) }\end{array}$ \\
\hline vftcav & MV & rf voltage on flattop cavity \\
\hline vmaincav1-vmaincav4 & MV & rf voltage on main cavity \\
\hline
\end{tabular}


[1] R. Dölling, Wire probe properties in Injector 2 and Ring cyclotron, Internal report identifier No. HIPA-BEAMDYDR84-023.02-030712 (unpublished).

[2] V. Smirnov, Computer codes for beam dynamics analysis of cyclotronlike accelerators, Phys. Rev. Accel. Beams 20, 124801 (2017).

[3] Y. J. Bi, A. Adelmann, R. Dölling, M. Humbel, W. Joho, M. Seidel, and T. J. Zhang, Towards quantitative simulations of high power proton cyclotrons, Phys. Rev. ST Accel. Beams 14, 054402 (2011).

[4] N. Pogue, A. Adelmann, and L. Stingelin, Opal simulations of the PSI ring cyclotron and a design for a higher order mode flat top cavity, in Proceedings of the International Particle Accelerator Conference (IPAC'17), Copenhagen, Denmark, 2017, International Particle Accelerator Conference No. 8 (JACoW, Geneva, Switzerland, 2017), pp. 3891-3894, https://doi.org/10.18429/JACoWIPAC2017-THPAB077.

[5] X. Huang and J. Safranek, Nonlinear dynamics optimization with particle swarm and genetic algorithms for SPEAR3 emittance upgrade, Nucl. Instrum. Methods Phys. Res., Sect. A 757, 48 (2014).

[6] X. Pang and L. Rybarcyk, Multi-objective particle swarm and genetic algorithm for the optimization of the LANSCE linac operation, Nucl. Instrum. Methods Phys. Res., Sect. A 741, 124 (2014).

[7] L. Yang, D. Robin, F. Sannibale, C. Steier, and W. Wan, Global optimization of an accelerator lattice using multiobjective genetic algorithms, Nucl. Instrum. Methods Phys. Res., Sect. A 609, 50 (2009).

[8] R. Husain and A. Ghodke, Constrained multi-objective optimization of storage ring lattices, Nucl. Instrum. Methods Phys. Res., Sect. A 883, 151 (2018).

[9] I. V. Bazarov and C. K. Sinclair, Multivariate optimization of a high brightness dc gun photoinjector, Phys. Rev. ST Accel. Beams 8, 034202 (2005).

[10] L. Yang, Y. Li, W. Guo, and S. Krinsky, Multiobjective optimization of dynamic aperture, Phys. Rev. ST Accel. Beams 14, 054001 (2011).

[11] A. Hofler, B.c. v. Terzić, M. Kramer, A. Zvezdin, V. Morozov, Y. Roblin, F. Lin, and C. Jarvis, Innovative applications of genetic algorithms to problems in accelerator physics, Phys. Rev. ST Accel. Beams 16, 010101 (2013).

[12] K. Deb, A. Pratap, S. Agarwal, and T. Meyarivan, A fast and elitist multiobjective genetic algorithm: NSGA-II, IEEE Trans. Evol. Comput. 6, 182 (2002).

[13] A. Adelmann, C. Baumgarten, M. Frey, A. Gsell, V. Rizzoglio, J. Snuverink (PSI), C. Metzger-Kraus, Y. Ineichen, S. Russell (LANL), C. Wang (CIAE), S. Sheehy, C. Rogers (RAL), and D. Winklehner (MIT), The OPAL (Object Oriented Parallel Accelerator Library) Framework, Technical Report No. PSI-PR-08-02, Paul Scherrer Institut, 2008-2019.

[14] C. Baumgarten, Beam based calibration measurements at the PSI cyclotron facility, in Proceedings of the International Conference on Cyclotrons and Their Applications (Cyclotrons'16), Zurich, Switzerland, 2016, International Conference on Cyclotrons and Their Applications No. 21 (JACoW, Geneva, Switzerland, 2017), pp. 342-344, http://jacow.org/cyclotrons2016/papers/thp16 .pdf.

[15] W. Joho, Recent and Future Developments at S.I.N., in Proceedings of the 8th International Conference on Cyclotrons and Their Applications, Bloomington, Indiana, 1978, International Conference on Cyclotrons and Their Applications No. 8 [IEEE Trans. Nucl. Sci. 26, 1950 (1979)], pp. 1950-1957, http://accelconf.web.cern.ch/AccelConf/ c78/papers/b-01.pdf.

[16] T. Stammbach, Experience with the high current operation of the PSI cyclotron facility, in Proceedings of the 13th International Cyclotron Conference, Vancouver, Canada, 1992, International Conference on Cyclotrons No. 13 (World Scientific, Singapore, 1993), pp. 28-35, http:// accelconf.web.cern.ch/AccelConf/c92/papers/i-04.pdf.

[17] S. Adam and W. Joho, Die Schaltung der Trimmspulen für das SIN $590 \mathrm{MeV}$ Ringzyklotron, 1974, https://doi.org/10 $.5281 /$ zenodo. 2556503 .

[18] A. Parfenova, C. Baumgarten, J. Humbel, A. Mezger, and A. Petrenko, Measurements of the beam phase response to correcting magnetic fields in PSI cyclotrons, in Proceedings of the International Particle Accelerator Conference (IPAC'16), Busan, Korea, 2016, International Particle Accelerator Conference No. 7 (JACoW, Geneva, Switzerland, 2016), pp. 1271-1273, https://doi.org/10.18429/ JACoW-IPAC2016-TUPMR019.

[19] E. Jones, T. Oliphant, P. Peterson et al., SciPy: Open source scientific tools for Python, 2018.

[20] A. Adelmann, P. Arbenz, and Y. Ineichen, A fast parallel Poisson solver on irregular domains applied to beam dynamics simulations, J. Comput. Phys. 229, 4554 (2010).

[21] Y. Ineichen, Toward massively parallel multi-objective optimization with application to particle accelerators, Ph.D. thesis, ETH Zurich, 2013.

[22] N. Neveu, L. Spentzouris, A. Adelmann, Y. Ineichen, A. Kolano, C. Metzger-Kraus, C. Bekas, A. Curioni, and P. Arbenz, A Parallel General Purpose Multi-Objective Optimization Framework, with Application to Beam Dynamics, arXiv:1302.2889.

[23] CSCS, https://www.cscs.ch/computers/piz-daint/, 2019.

[24] H. Ishibuchi, Y. Nojima, and Tsutomu Doi, Comparison between single-objective and multi-objective genetic algorithms: Performance comparison and performance measures, in Proceedings of the 2006 IEEE International Conference on Evolutionary Computation (IEEE, New York, 2006), pp. 1143-1150, https://doi.org/10 $.1109 /$ CEC.2006.1688438.

[25] J. R. Alonso and DAESALUS Collaboration, High current $\mathrm{H}_{+}^{2}$ cyclotrons for neutrino physics: The IsoDAR and DAESALUS projects, AIP Conf. Proc. 1525, 480 (2013).

[26] A. Adelmann and DAESALUS Collaboration, Modelling challenges of the high power cyclotrons for the DAESALUS project, AIP Conf. Proc. 1560, 709 (2013).

Correction: The previously published Figure 10 contained errors in the $x$-axis scales for trim coils $1,2,4-11,13,14$, and 18 . These errors have been fixed. 